\title{
Belphégor
}

\section{De l'école à la maison à la cour de récréation. Métamorphoses et disparition des illustrés pour petites filles (1946-1974)}

\section{Béatrice Guillier}

\section{(2) OpenEdition}

Journals

Édition électronique

URL : https://journals.openedition.org/belphegor/4250

DOI : 10.4000/belphegor.4250

ISSN : 1499-7185

Éditeur

LPCM

Référence électronique

Béatrice Guillier, «De l'école à la maison à la cour de récréation. Métamorphoses et disparition des illustrés pour petites filles (1946-1974) », Belphégor [En ligne], 19-2 | 2021, mis en ligne le 06 janvier 2022, consulté le 20 septembre 2022. URL : http://journals.openedition.org/belphegor/4250 ; DOI : https://doi.org/10.4000/belphegor.4250

Ce document a été généré automatiquement le 20 septembre 2022.

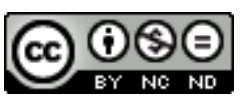

Creative Commons - Attribution - Pas d'Utilisation Commerciale - Pas de Modification 4.0 International - CC BY-NC-ND 4.0

https://creativecommons.org/licenses/by-nc-nd/4.0/ 


\title{
De l'école à la maison à la cour de récréation. Métamorphoses et disparition des illustrés pour petites filles (1946-1974)
}

\author{
Béatrice Guillier
}

Les années 1960 et 1970 ont vu les publications pour petites filles disparaître les unes après les autres. La Semaine de Suzette, modèle du genre depuis 1905, a mis la clé sous la porte en 1960. Fillette Jeune Fille fut le suivant à disparaître, en 1964, tandis que Lisette et Nade n'ont pas survécu aux années 1970. Le contexte social et culturel est jugé rétrospectivement impitoyable pour ces journaux d'un autre temps. «Le journal est tombé, il fallait qu'il tombe, c'était plus d'époque ", confie une illustratrice chez Suzette, en 20161. D'aucuns pourraient croire que la disparition de ces périodiques était « inévitable ». Mais si de nombreux signes annonçaient la crise généralisée de ces hebdomadaires jugés dépassés (baisse des ventes, instabilité de la maquette et des contenus, concurrence des journaux adolescents), leur identité de publications féminines ne semblait pas devoir nécessairement les mener à leur perte. Malgré la généralisation de la mixité scolaire et l'influence de la seconde vague du féminisme, force est en effet de constater que les magazines féminins se maintiennent et constituent une part importante du marché de nos jours. Ce sont uniquement les journaux pour petites filles ${ }^{2}$ qui ne prennent pas le tournant des années 1970, et ce alors même que la période voit le développement d'un marketing de plus en plus genré à destination des enfants, notamment dans le domaine du jouet. Comment penser cette disparition? Pourquoi le périodique pour petites filles devient-il plus indésirable qu'un numéro de Marie-Claire ou qu'un poupon en plastique habillé de rose et destiné à des "petites mamans»? Quels éléments permettent d'expliquer cette transition dans l'après-guerre français d'une presse pour fillettes à des magazines conjugués au masculin, envisagé comme « neutre universel »? 
2 Si les illustrés pour petites filles ont fait l'objet de quelques études monographiques concernant la période d'avant-guerre ${ }^{3}$, les décennies 1950 et 1960 n'ont pas été étudiées en profondeur. Les recherches sur la presse jeunesse de ces années-ci se sont focalisées sur les mensuels pour adolescents comme Salut les Copains ou Mademoiselle Âge Tendre qui ont été lancés dans les années 1960, participant ainsi à l'émergence de ce que l'on a appelé la «culture jeune $»^{4}$. Quant à la question de la mixité, amplement traitée en histoire sociale, elle n'a pas encore donné lieu à une étude spécifique des représentations de cette période de bouleversement de l'enseignement primaire et secondaire dans les médias pour petites filles.

3 C'est dans ce cadre que j'ai décidé d'étudier la disparition des journaux pour petites filles à travers l'analyse d'un corpus d'illustrés, en me concentrant sur les espaces éducatifs de ces journaux. Lorsque l'on feuillette La Semaine de Suzette (1905-1960), Fillette, devenue Fillette Jeune Fille (1909-1964), Bernadette alias Nade (1914-1972) et Lisette (1921-1974), on trouve dans ces journaux des rubriques d'apprentissage qui constituent des objets d'étude précieux lorsque l'on s'intéresse à cette presse à caractère éducatif. J'ai procédé à la recension et à l'analyse intégrale des rubriques de ces journaux sur une période de près de trente ans, ce qui m'a amenée à formuler l'hypothèse que, plutôt que d'être de simples marchandises destinées au "marché des petites filles», les hebdomadaires pour jeunes filles se situent dans un nexus éducatif plus large, allant de l'école à l'apprentissage familial. Avec les transformations des années 1960-1970 et l'émergence de la jeunesse comme classe d'âge reconnue socialement, ainsi que l'arrivée de la mixité scolaire comme norme et comme réalité sociale, ces journaux ne parviennent plus à s'inscrire dans le nouveau contexte socioculturel de la France de la seconde moitié du $\mathrm{xx}^{\mathrm{e}}$ siècle. Après plusieurs tentatives de réinvention et l'adoption de diverses stratégies de survie, ils finissent par disparaître. Afin de tenter de comprendre les raisons de cette disparition, je vais étudier la période qui va de l'autorisation de reparution des quatre illustrés après la guerre, en 1946, jusqu'à la disparition du dernier d'entre eux, Lisette, en 1974, à la veille de la loi Haby de 1975 qui généralise la mixité dans tous les degrés de l'enseignement.

4 Je reviendrai d'abord sur les liens historiques entre les illustrés et l'école, et plus largement sur la nature pédagogique qui marque les périodiques jusqu'à la Seconde Guerre mondiale. Je poursuivrai par une étude de la manière dont la mixité scolaire fait son apparition comme thème dans les journaux. Je présenterai ensuite une analyse du recul des savoir-faire et de l'apprentissage technique liés à la sphère domestique, au profit du savoir-être par la consommation et le loisir. En conclusion, je montrerai que la chute des périodiques pour petites filles peut s'expliquer par la diffusion institutionnelle de la vision d'une enfance comme âge de la neutralité et de l'indifférenciation sexuelle.

\section{Pédagogie par l'image et illustrés}

5 La presse pour enfants connaît un essor important dans la seconde moitié du XIX siècle, du fait de l'alphabétisation croissante de la population, y compris féminine, sous l'effet des grandes lois sur l'enseignement primaire. Cette mutation est une aubaine pour les éditeurs : un arrêté de janvier 1890 stipule que chaque enfant scolarisé doit posséder six manuels ${ }^{5}$. Passé depuis 1870 dans le régime de la libre concurrence, le système éditorial français connaît des heures fastes. Outre les manuels scolaires, des romans 
pour enfants sont régulièrement publiés. Les éditeurs désireux de tester leur popularité créent des titres de presse pour faire paraître ces romans en feuilleton, à tel point qu'entre 1857 et 1904, quarante journaux pour la jeunesse sont créés en France ${ }^{6}$.

À partir de 1880, l'image s'impose dans l'univers de l'enfant scolarisé, avec l'introduction dans les programmes officiels de l'enseignement dit " par l'aspect », une forme de pédagogie par l'image ${ }^{7}$. Le ministère de l'Instruction publique crée une commission de la décoration des écoles et de l'imagerie scolaire, afin de donner aux éditeurs des consignes sur la publication des images, notamment sur l'importance d'adapter le type de l'image à l'âge des enfants auxquels elle est destinée ${ }^{8}$.

7 L'imprimé n'échappe pas à ce tournant de l'image pédagogique, et les titres de presse enfantine donnent une place prépondérante à l'illustration dans les fictions comme dans les rubriques instructives. En 1899, Armand Colin publie Le Petit Français illustré, qui est suivi en 1903 de La Jeunesse illustrée de Fayard9. En 1904, les éditions Offenstadt publient le Petit Illustré, qui contribue également à la popularisation des publications hebdomadaires dotées d'une riche iconographie pour la jeunesse ${ }^{10}$. Les journaux pour petites filles, comptant déjà quelques grands titres comme Le Journal des demoiselles (1833-1922) ou Le Magasin des demoiselles (1844-1896), adoptent au tout début du $\mathrm{xx}^{\mathrm{e}}$ siècle ce nouveau format qui mêle divertissement et apprentissage fondé sur l'image ${ }^{11}$. Les gravures et dessins y sont moins des illustrations que des exempla, « une ressource rhétorique dans un discours de persuasion, un modèle à suivre pour l'écolier ${ }^{12}$ ». Un même objectif d'éducation par l'image caractérise alors un ensemble d'artefacts destinés à l'enfance: manuels scolaires, planches didactiques, images d'Épinal et périodiques ${ }^{13}$.

8 Dans une continuité formelle avec les nouvelles formes pédagogiques, ces illustrés du début du siècle paraissent le jeudi ou le dimanche, jours chômés pour les petits écoliers, et viennent compléter les apprentissages scolaires. Dès son premier numéro, daté du 17 juillet 1921, on trouve dans l'éditorial de Lisette la promesse suivante : «[Lisette] vous enseignera mille petits secrets qui feront de vous, dans le présent, la joie de vos chers parents et, plus tard, des femmes ingénieuses, vaillantes et bonnes qui sauront mériter le bonheur ". Dans la lignée des manuels de travaux amateurs, très populaires à partir de la seconde moitié du XIX ${ }^{\mathrm{e}}$ siècle $^{14}$, Lisette, Suzette, Fillette et les autres proposent des activités manuelles à exercer dans le cadre domestique. Les activités d'entretien sont encouragées, du fait de la diffusion d'un idéal bourgeois du foyer où la figure de l'épouse est centrale. Une même valorisation se retrouve dans les manuels d'enseignement ménager, qui existent depuis la fin du xix ${ }^{\mathrm{e}}$ siècle, et qui partagent avec les périodiques des codes narratifs : l'initiation passe souvent par des dialogues fictifs entre une jeune femme et une femme plus expérimentée qui lui transmet son savoirfaire ${ }^{15}$. Dans les illustrés, ce sont des figures tutélaires qui se chargent d'instruire les lectrices. Marraine, Tante Biscuit, Tante Conseil ou $\mathrm{M}^{\text {lle }}$ Passe-temps se succèdent auprès des fillettes pour leur apprendre à cuisiner, coudre, bien se tenir, soigner un rhume ou allonger leur silhouette grâce à la gymnastique. Les rubriques de travaux d'aiguille, richement illustrées, occupent une place particulièrement centrale, juxtaposant une iconographie proche de celle des catalogues de mode et des textes encourageant les lectrices à coudre et à tricoter pour leurs proches ou leur poupée (ill. 1). 
III.1. "Le tricot pour ma petite sœur », Lisette, $1^{\mathrm{er}}$ janvier 1922, p.11. Domaine public, Gallica [En ligne], URL : https://gallica.bnf.fr/ark:/12148/bpt6k9114944v.

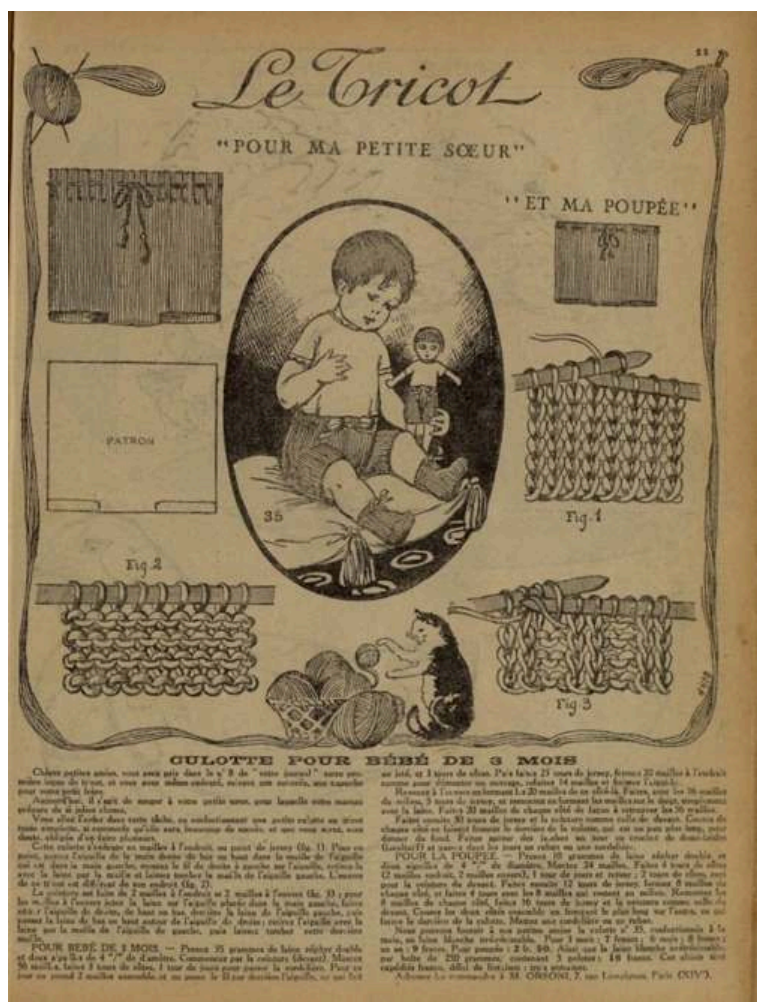

Suzette, Lisette, Bernadette et même la tant décriée Fillette ${ }^{16}$ affichent des objectifs qui les classent du côté de la presse éducative, malgré la présence de fictions illustrées (lesquelles véhiculent autrement l'idéologie du journal). Alain Fourment analyse le tournant que prend la presse jeunesse dans les années 1930, avec l'arrivée massive des productions américaines sur le marché français, comme une victoire de la presse de divertissement sur la presse éducative ${ }^{17}$. Pourtant, les illustrés pour petites filles ne montrent pas la même perméabilité aux bandes dessinées d'action que leurs équivalents masculins, s'inscrivant davantage dans une forme de conservatisme par rapport aux publications $\mathrm{du} \mathrm{XIX}^{\mathrm{e}}$ siècle. Ils bénéficient d'une production entièrement française et ne publient pas les histoires étrangères traduites à la va-vite que proposent les journaux pour garçons. Leur maquette et leur rubriquage évoluent très peu avant la Seconde Guerre mondiale, et il faut attendre la fin des années 1950 pour percevoir des changements majeurs dans leurs contenus et leurs discours. Ces éléments indiquent le refus de ces objets de se placer résolument dans la sphère du divertissement et du loisir. De plus, leurs résistances aux dynamiques qui dominent le marché de l'époque qui valorise alors la bande dessinée étrangère - les éloignent du domaine des marchandises pour les situer dans une continuité avec l'école et le cadre familial.

La généralisation de la presse de divertissement rencontre par ailleurs un fort rejet dans l'opinion publique. Des censeurs, dont l'abbé Bethléem dans sa Revue des lectures, la refusent en bloc. Témoignant de ces réticences, la loi de 1949 fait de la France le premier pays au monde à posséder un régime légal spécifique pour ses publications jeunesse. Elle met en lumière le refus de soutenir sur le plan institutionnel des titres populaires et divertissants, au profit d'une presse morale et éducative, surveillée par l'État. Dans la période d'après-guerre où les journaux pour petites filles relayent le discours nataliste et hygiéniste de l'État, discours qui passe notamment par une 
valorisation des compétences ménagères, les illustrés pour petites filles rencontrent l'idéal français d'une presse jeunesse éducative. Dans leur forme et dans le contenu de leurs rubriques, du fait de leur statut légal et de leur rythme de parution, les périodiques pour petites filles sont pensés dans leur articulation avec l'école. Ils s'inscrivent dans un ensemble d'objets et d'institutions qui peuplent l'espace éducatif et socialisateur des "futures mères" (ill. 2). Cette approche renvoie à la théorie de Colette Guillaumin sur l'appropriation des femmes: le corps féminin, soit ses compétences reproductives et son travail domestique, est alors appréhendé comme la propriété collective des hommes et des institutions étatiques ${ }^{18}$. Bien que les journaux ne possèdent aucun lien effectif avec les organismes publics, c'est bien cette appropriation que manifeste leur parfaite congruence avec des politiques nationales de contrôle et de formation des femmes. Les lois scolaires et l'enseignement ménager viennent contribuer à une organisation du foyer fondée sur le dimorphisme sexuel. Ils visent à produire une mère éduquée qui transmet les valeurs nationales à ses enfants ${ }^{19}$. On assiste dans la première moitié $\mathrm{du} \mathrm{xx}^{\mathrm{e}}$ siècle à ce que Françoise Thébaud a appelé «la nationalisation des femmes ${ }^{20}$ », ces dernières étant investies par l'État d'une mission éducative en tant que mères. Les filles sont incitées à adopter une forme d'abnégation au service de leur famille, mais également de leur patrie ${ }^{21}$. Les mères doivent enfanter de futurs citoyens et citoyennes, dont les moindres déviances leur seront immédiatement reprochées ${ }^{22}$. Les périodiques pour petites filles participent à la construction d'une future mère de famille patriote, en transmettant une idéologie impérialiste et une vision d'un monde ordonné et hiérarchisé en termes de classes ${ }^{23}$.

III.2. "Une grande sœur modèle ", Lisette Télé Jeune, Éditions de Montsouris, 5 mars 1972. Droits réservés.

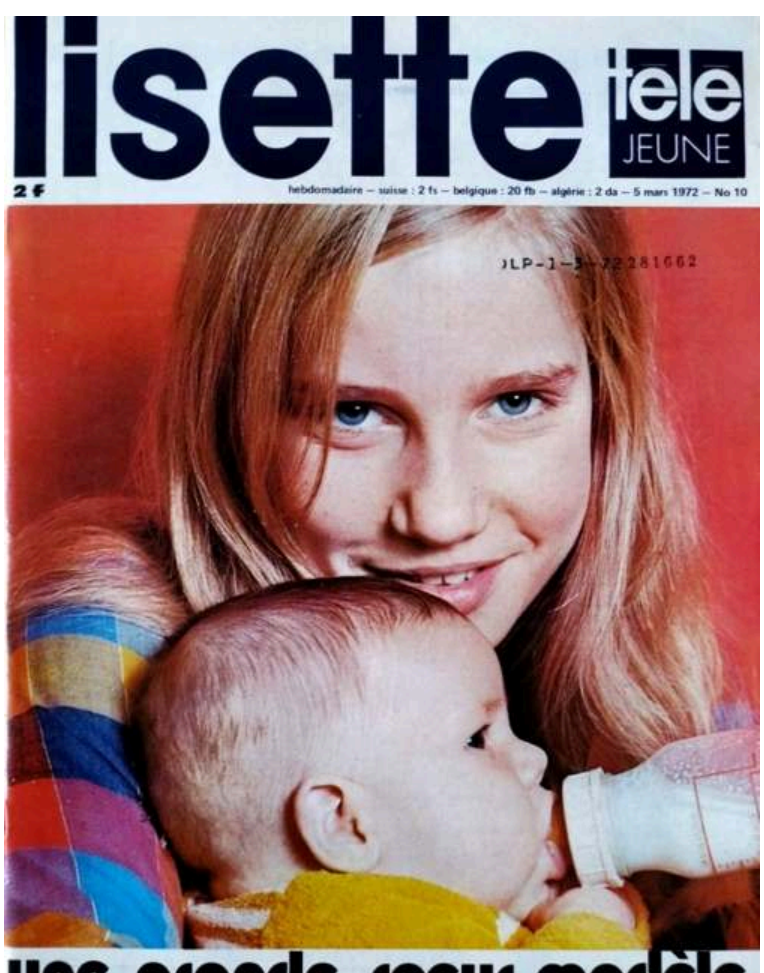

Une grande roeur modele 


\section{Éduquer les filles, excuser les garçons}

11 Après l'interruption suscitée par la guerre, les illustrés pour petites filles obtiennent sans difficulté le droit de reparaître en 1946, tout en s'inscrivant par la suite dans le cadre énoncé par la loi de moralisation de 1949. Ces journaux vont rapidement se saisir d'un sujet central chez les professionnels de l'éducation dans les années 1950 : la mixité scolaire. Les journaux pour petites filles documentent le long processus qui va mener à la loi Haby de 1975, avec d'autant plus d'acuité que la mixité engage, leurs rédacteurs le devinent, leur existence même. Les années 1960 voient en effet se multiplier les publications mensuelles mixtes à grand tirage qui créent une concurrence en ciblant la part la plus âgée des lectrices des illustrés. Cette confrontation pousse les illustrés à se saisir de la question de la mixité et à moderniser leur image pour continuer à se vendre. Ce sont ces stratégies d'adaptation face à des bouleversements socio-économiques majeurs qui font de ces journaux un matériau privilégié pour comprendre les changements survenus dans la vie des lectrices.

12 À partir de la fin des années 1950, les garçons investissent l'espace des journaux pour petites filles, en tant que chroniqueurs, lecteurs et interlocuteurs. En 1969, Lisette accepte de faire l'intermédiaire entre des correspondants garçons et filles. Les lecteurs gagnent également une place dans le courrier des lectrices, en tant que correspondants mais aussi en tant que sujets de préoccupation. Le thème de la cohabitation avec les garçons divise : Geneviève V. revendique dans le Lisette du 29 janvier 1969 : « Moi, je ne suis pas pour les écoles mixtes. Ah! non, alors. Les garçons, ça chahute toujours ». Le principal sujet de discorde concerne la brutalité, la saleté et les moqueries des garçons, que les filles perçoivent comme un obstacle au bon déroulement de leurs études (ill. 3). Face à ces réclamations, les réponses de Marraine aux courriers dédramatisent les actions des garçons. Les fillettes se retrouvent souvent blâmées dans leur refus de comprendre les attitudes de leurs nouveaux camarades de classe et de leur pardonner certains écarts de conduite. Aux petites filles qui se plaignent de se faire siffler par un groupe de garçons lorsqu'elles passent en bicyclette, on répond: "Peut-être ont-ils envie de faire votre connaissance? » (Lisette Télé Jeune, 14 août 1971). La généralisation de la coéducation s'accompagne donc dans l'espace des journaux de leur cohabitation dans la rubrique du courrier. 
III.3. "Être fraîche et jolie... Mais les garçons ? ", Lisette et Caroline, Société Française de Presse Illustrée, août 1973. Droits réservés.

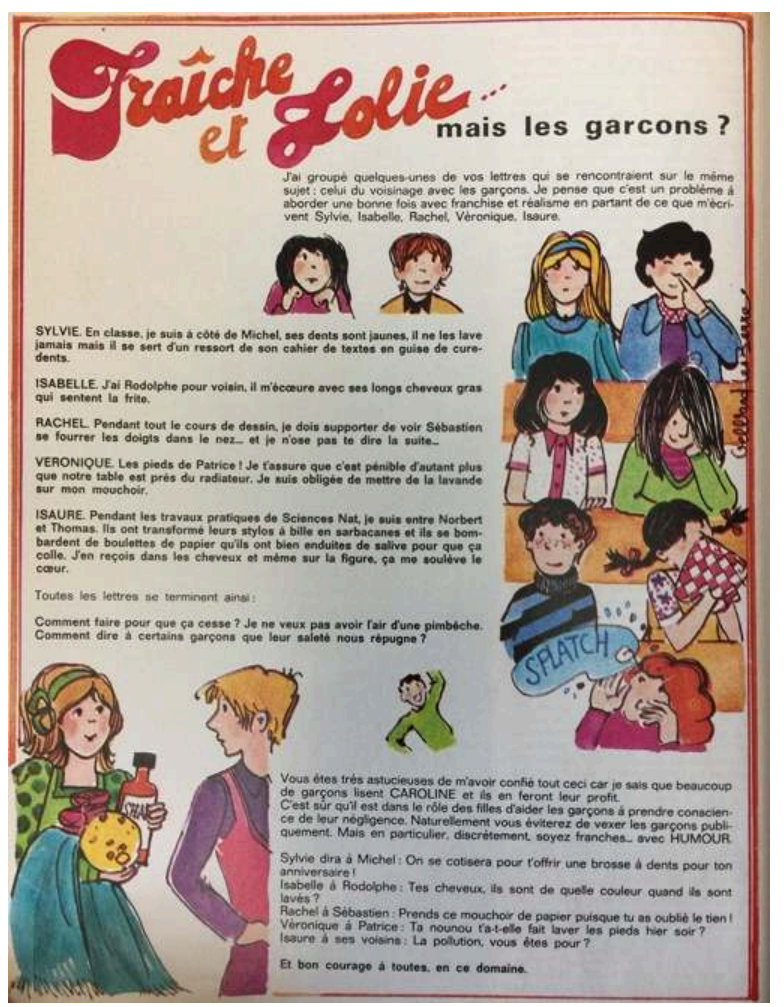

13 Si la question de la coéducation avait été débattue à la fin du XIX ${ }^{e}$ siècle et au début du $\mathrm{xx}^{\mathrm{e}}$ siècle par les théoriciens de l'éducation, la généralisation de la mixité scolaire après la Seconde Guerre mondiale a lieu pour des raisons économiques. Elle permet de rassembler dans les mêmes classes et établissements les deux populations, qui connaissent une augmentation démographique importante ${ }^{24}$. Les justifications éducatives viennent dans un second temps. À partir des années 1960, les réponses au courrier des lectrices des hebdomadaires commencent à présenter plusieurs arguments favorables à la cohabitation des écoliers et écolières, justement parce que les deux catégories d'individus seraient diamétralement opposées. En 1969, Marraine conseille à une lectrice de se mêler aux jeux des garçons à la récréation: "Ta confiance les amènera peu à peu à se... civiliser. À mon avis l'école mixte a un grand avantage : elle aguerrit les filles et adoucit les garçons » (Lisette, 29 janvier 1969). Les filles doivent ainsi prendre en charge l'éducation de leurs condisciples. Luce qui se plaint des jeux violents auxquels se livrent ses petits voisins se voit conseiller de les inviter à jouer avec elle : «Le monopoly, les cartes permettraient à tes remuants camarades de te livrer de rudes batailles... sans trop t'effrayer: Et peut être aimeraient-ils beaucoup cette autre manière de se faire la guerre » (Lisette Télé Jeune, 19 novembre 1972).

Les administrateurs de l'Éducation nationale ne disent pas autre chose lorsqu'ils se penchent sur la question en 1965 : l'école mixte apprend à connaître l'autre sexe dans une société dans laquelle leur cohabitation est de plus en plus importante ; l'école mixte serait donc une conséquence naturelle des bouleversements socio-économiques de l'après-guerre ${ }^{25}$. Dans le Lisette Télé Jeune du 19 juin 1971, Régine, qui prend la suite de Marraine comme interlocutrice privilégiée des lectrices, rassure Nadine sur la normalité de sa camaraderie avec un garçon et emploie cet argument : "Cette gêne est un reste de l'éducation d'autrefois - filles d'un côté, garçons de l'autre - et elle nous 
marque tous plus ou moins. C'est ce qui explique que la mixité, pourtant si naturelle, soulève parfois tant de questions ». En relayant avec zèle les discours officiels sur la mixité scolaire, les périodiques se situent une fois de plus dans la continuité des politiques publiques en matière d'éducation. Les rédactions se livrent ainsi à un périlleux exercice d'équilibriste, partagées entre leur rôle traditionnel de soutien aux institutions et donc à la mixité scolaire, et leur volonté de plaire à des lectrices parfois sceptiques face à cette réforme.

\section{Pimbêches et garçons manqués}

Cette prise de position, si elle est générale parmi les illustrés pour petites filles, ne se traduit pas par les mêmes imageries et discours. Ceux-ci diffèrent selon les stratégies de survie adoptées par des journaux qui assistent à la baisse dramatique de leurs chiffres de vente au cours des années $1950^{26}$. Lisette manifeste une position pro-mixité très claire dans ses espaces de communication avec ses lectrices, et défend dans l'ensemble une vision plutôt apaisée des rapports filles/garçons, malgré les courriers parfois agacés des fillettes et quelques rubriques au ton humoristique.

De son côté, Bernadette (Nade à compter de 1964) assume tout au long de la période la mise en avant d'une mixité de fait, particulièrement à travers ses couvertures représentant souvent des groupes d'enfants des deux sexes partageant une même activité (ill. 4). Le journal, d'obédience catholique, appartient à la Maison de la Bonne Presse, qui devient Bayard Presse en 1969; la maison d'édition semble affirmer, dès l'après-guerre, une volonté de développer une presse éducative ${ }^{27}$ et mixte, et donc de ne pas s'appesantir sur d'éventuels conflits filles/garçons.

III.4. Nade, Maison de la Bonne Presse, 22 janvier 1967. Droits réservés.

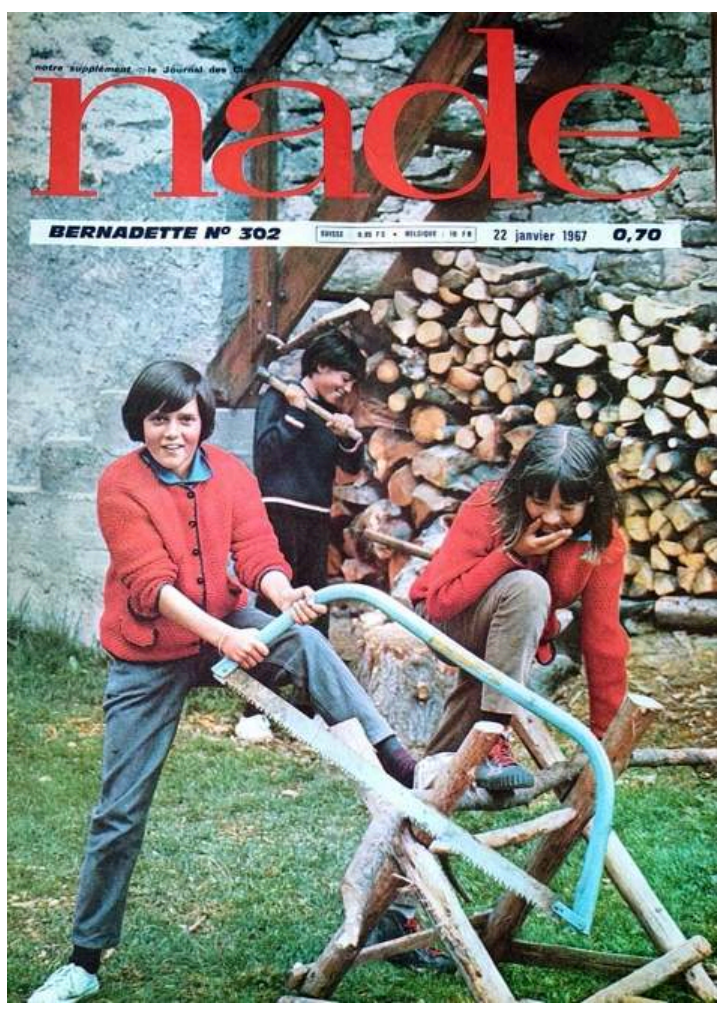



mise en scène d'un antagonisme taquin entre filles et garçons, notamment à travers sa série de couvertures « Les garçons sont... » (ill. 5) : les garçons sont coquets, maladroits, douillets, paresseux... On y voit des jeunes garçons en pleine action, en train de se regarder dans un miroir, d'arborer un énorme pansement autour de la tête ou de faire tomber une pile d'assiettes. Les photographies sont ouvertement comiques : les poses y sont outrées, les mimiques appuyées, afin de souligner le côté absurde des stéréotypes qu'elles illustrent. Ces idées reçues n'en sont d'ailleurs pas: les situations semblent davantage relever d'une inversion des stéréotypes, puisqu'une partie de ces défauts sont plutôt considérés comme féminins. Cette approche humoristique renvoie dos à dos garçons et filles, sans prendre position, comme la bande dessinée Paul et Mic que l'on trouve chaque semaine dans le journal à compter de 1961 et qui met en scène deux jeunes qui se disputent à propos de leurs irréductibles différences, liées à leur genre. Après une brève incursion en 1964 du côté de la presse mixte avec un nouveau journal qui s'intitule comme ces nouveaux héros, Paul et Mic, Fillette Jeune Fille adopte définitivement ses nouveaux atours en 1965 quand elle devient Quinze ans, confirmant sa volonté de toucher un public féminin plus âgé.

III.5. « Les garçons sont maladroits ", Fillette Jeune Fille, Société parisienne d'édition, 14 décembre 1960. Droits réservés.

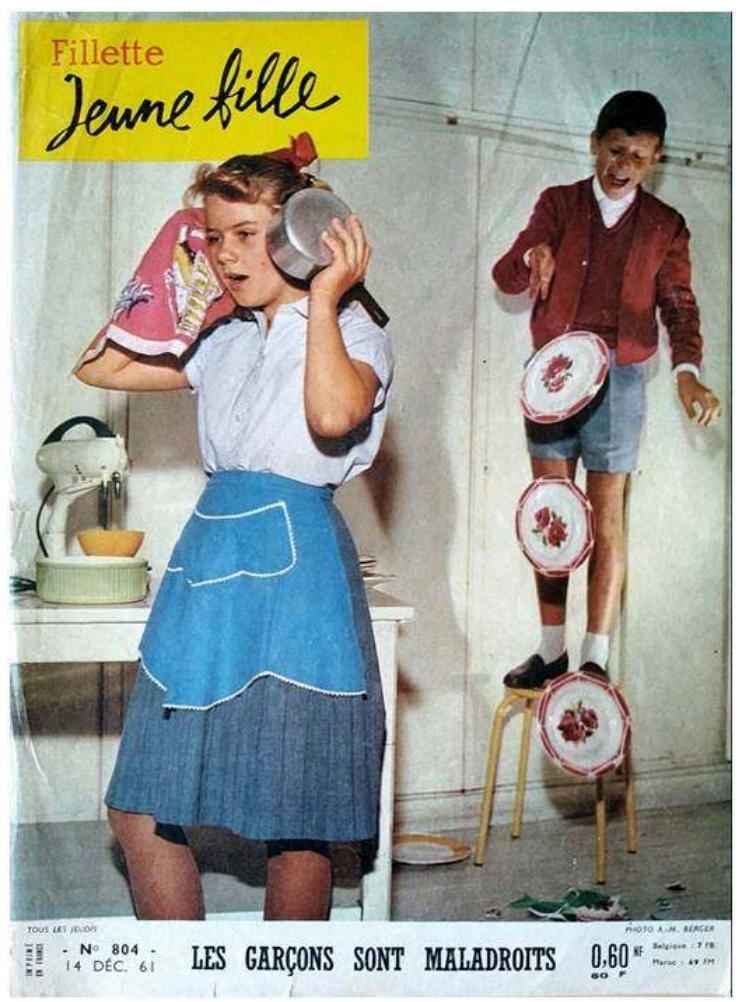

Quant à La Semaine de Suzette, l'illustré disparaît dès 1960 après avoir mis en scène un conflit filles/garçons, tout en reconnaissant la primeur à donner à présent à la mixité : l'abonnement des petites Suzette est d'ailleurs automatiquement transformé en abonnement au Journal de Mickey.

19 Mis à part Nade, les périodiques pour petites filles développent une double narration: celle de la mixité scolaire précieuse pour apprendre à se connaître, et celle des conflits filles/garçons dus à leurs différences. Cette fracture devient un ressort comique des 
hebdomadaires pour petites filles, qui donnent la parole à des jeunes garçons fictifs. Jean-Loup débarque ainsi avec fracas dans La Semaine de Suzette en 1958. Ce jeune garçon dont on retrouve la frimousse chaque semaine anime successivement trois rubriques : "Votre ami Jean-Loup ", "Allô allô ici Jean-Loup » et «Le club des JeanLoup ». Il y fait part de ses déboires avec les filles, au premier rang desquelles se trouvent ses sœurs et ses cousines (ill. 6). Jean-Loup donne son avis sur l'éducation prodiguée aux enfants, sur l'habillement et les postures féminines, et propose des tests de personnalité biaisés aux petites lectrices. Invitées à répondre à une série de questions afin de savoir si elles présentent des traits de caractère traditionnellement attribués aux femmes, comme la curiosité ou l'admiration pour les stars, les fillettes ne sont confrontées qu'à des résultats hostiles : soit elles se conforment aux stéréotypes, soit elles mentent nécessairement, selon Jean-Loup. Le jeune garçon reproche ainsi continuellement aux filles les caractères stéréotypés qu'il leur attribue, mais s'emporte contre les filles qu'il considère comme des garçons manqués, jusqu'à user de violence physique envers elles : «J'aime mieux vous dire que j'étais furieux. Alors, puisque cette fille voulait jouer au garçon manqué, je me suis arrangé pour la faire tomber. Histoire de vérifier son héroïsme » (La Semaine de Suzette, 27 février 1958).

III. 6. «Allô allô, Ici Jean-Loup : Les filles ne savent pas ce qu'elles veulent ! », La Semaine de Suzette, 6 mars 1958. Droits réservés.

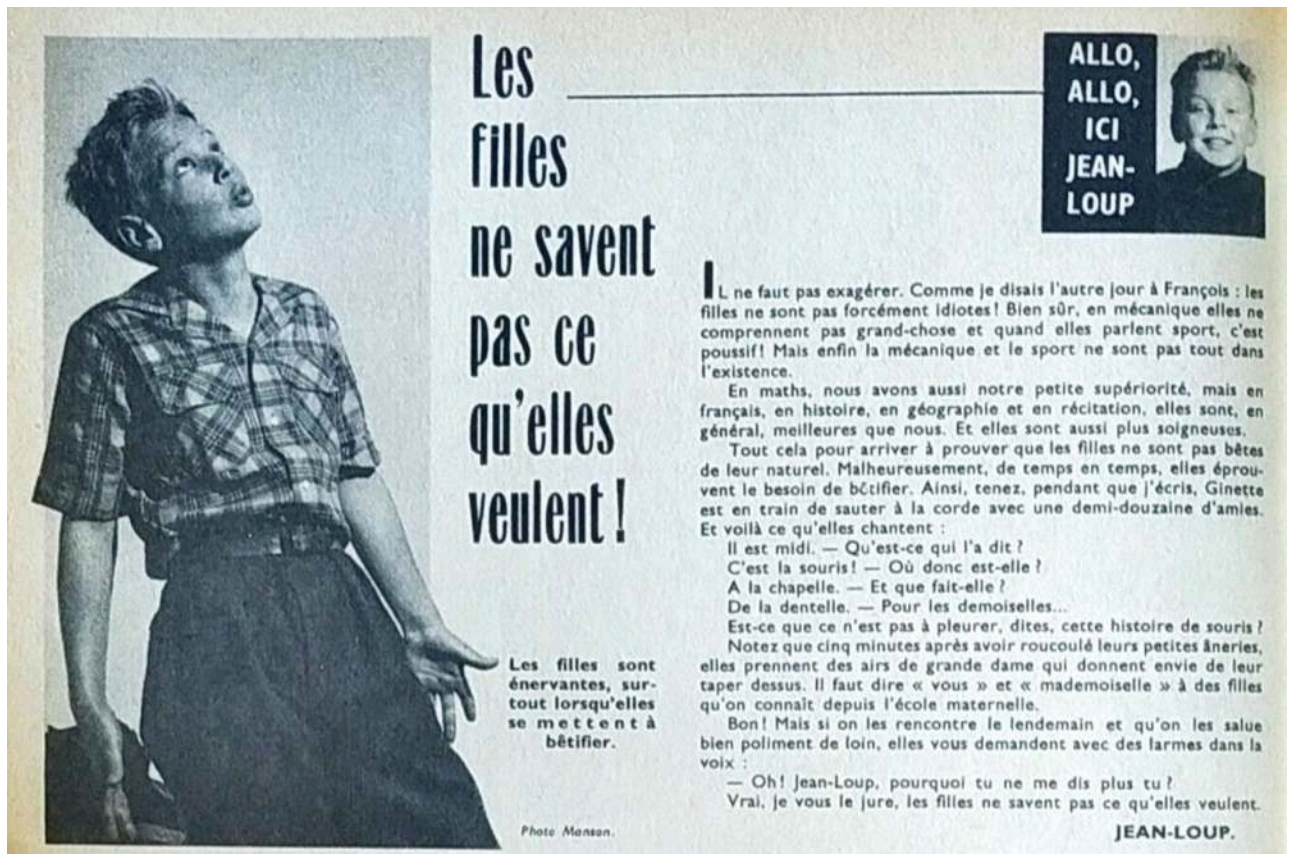

Cette hostilité se retrouve chez Aimé O. dans Fillette Jeune Fille entre 1960 et 1961 . Ce jeune garçon, dont on ne connaît le visage que par un dessin crayonné en noir et blanc, envoie chaque semaine une lettre reproduite dans le journal en écriture cursive pour créer un effet de réel. Il se plaint de problèmes semblables à ceux de Jean-Loup : des figures féminines familiales agaçantes et des déboires personnels, toujours à mettre sur le compte d'une irréductible différence entre garçons et filles. On retrouve une même violence verbale, qui repose sur l'usage d'un registre de langue et d'un vocabulaire appartenant a priori à un locuteur plus âgé, ainsi que la présence de menaces d'ordre physique. Le dispositif est complexifié par un droit de réponse donné dans le journal (ill. 7). Des interlocutrices, qu'on peut supposer fictives, se joignent à la conversation : 
d'abord Virginie, qui répond vertement aux allégations d'Aimé O., puis Sophia, qui dans le Fillette Jeune Fille du 23 février 1961 apporte son soutien au garçon : «Bravo Aimé 0.! Peu de garçons écrivent avec autant de franchise que vous [...]. Nous admirons votre personnalité et la façon spirituelle avec laquelle vous criez leur fait aux pimbêches ». Introduisant ces échanges hebdomadaires, la rédaction se revendique «impartiale », et salue le « joli minois » de Virginie comme de Sophia.

III.7. « Aimé O. », Fillette Jeune Fille, Société parisienne d'édition, 11 août 1960, p. 30. Droits réservés.

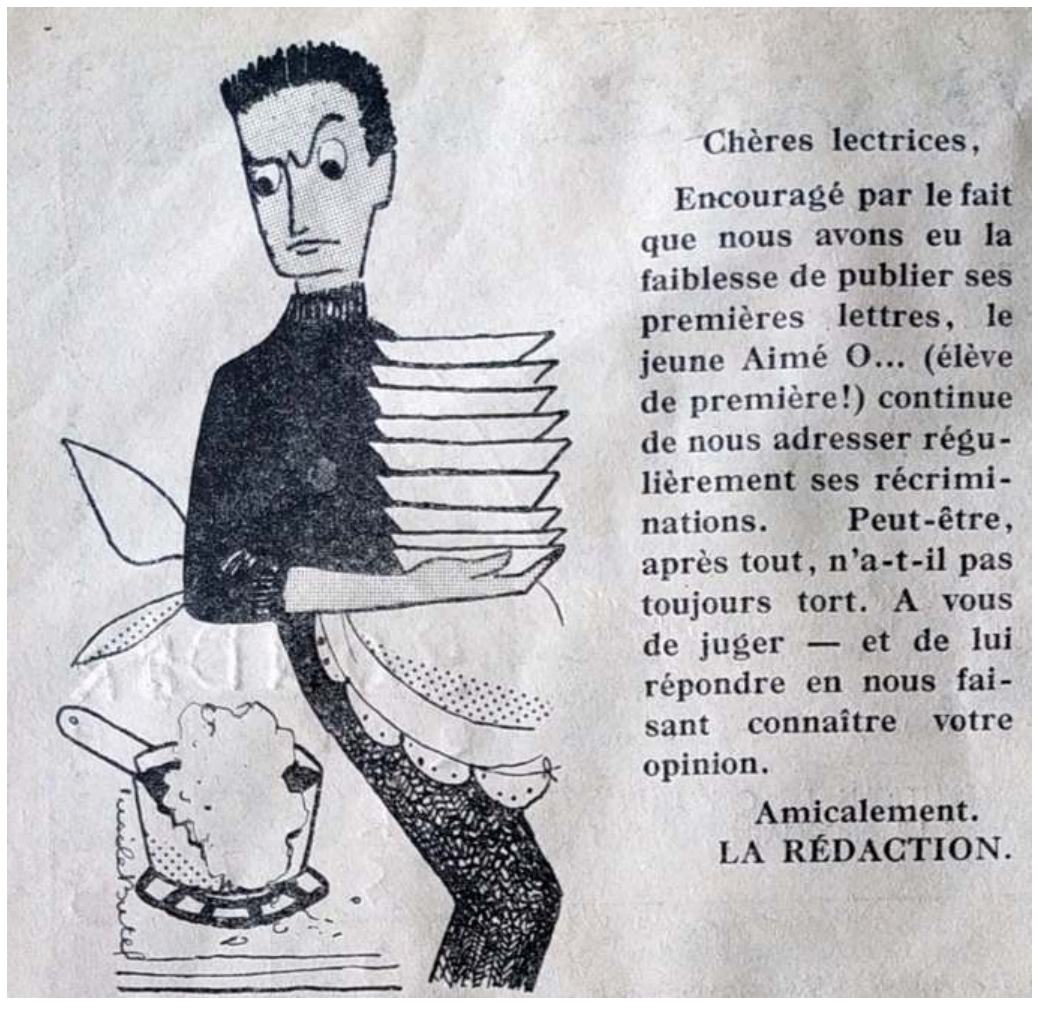

Qu'il s'agisse de Jean-Loup ou d'Aimé O, les rubriques d'échanges fictifs figent un ensemble de normes de genre, sur un registre brutal qui détonne avec le ton employé par ailleurs dans les publications pour petites filles. Les lectrices, qui signalent parfois dans le courrier leur goût pour ces joutes verbales et ces personnages hauts en couleurs, se retrouvent critiquées par leurs pairs dans les pages d'une publication qui leur était auparavant réservée.

Si les garçons s'exprimant dans le courrier des lecteurs sont généralement beaucoup plus amicaux, les jeunes choisis par Lisette pour le numéro spécial garçons du 2 mars 1969 tiennent des discours similaires à ceux de Jean-Loup et d'Aimé O. Dans une rubrique intitulée "À cœur ouvert » qui occupe une pleine page dans ce numéro (ill. 8), les garçons reviennent sur leurs goûts et dégoûts concernant les filles. Cette page concentre toutes les revendications portées par les figures masculines des journaux depuis plusieurs années, et constitue donc un condensé de tous les stéréotypes associés aux petites filles. 
III. 8. «À cœur ouvert », Lisette, Éditions de Montsouris, 2 mars 1969, p. 15. Droits réservés.

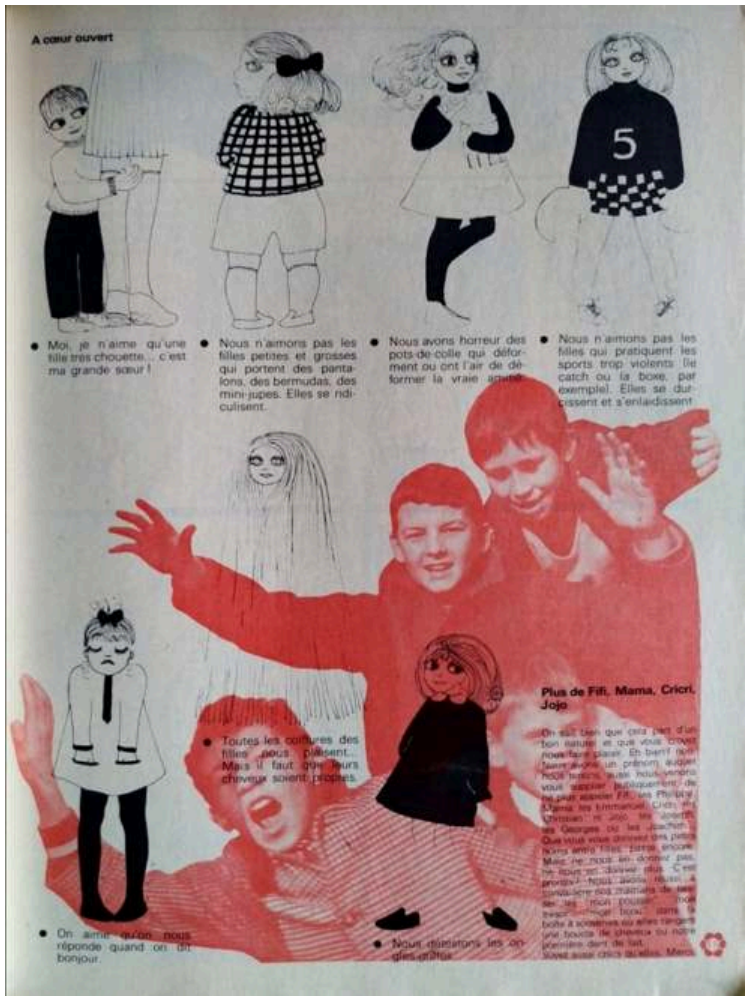

Ces dispositifs, qui tranchent à première vue avec l'ethos de pacificateur adopté par ces journaux, s'inscrivent dans l'idéologie présente dans les discours institutionnels sur la mixité. Les garçons et les filles sont fondamentalement différents, et il faut apprendre à se connaître pour cohabiter en bonne intelligence ; l'école mixte remplit ce rôle, et les périodiques pour petites filles également, en relayant les paroles de garçons imaginaires ou réels. Il s'agit donc à la fois d'exacerber les différences et de les surmonter, dans un discours qui peut paraître contradictoire et qui est décrit par Ros Ballaster et ses coautrices dans Women's World, à propos de la presse féminine : «there is an evident tension between the need to confirm the centrality and desirability of men in all women's lives and the equally insistent recognition of men as a problem for and threat to women ${ }^{28} »$. Les illustrés pour petites filles relèvent d'une idéologie patriarcale, et incitent leurs lectrices à apprendre le futur rôle social qu'elles occuperont en tant que femmes. La généralisation de la mixité scolaire et l'arrivée des garçons dans les pages des journaux provoquent un changement dans le type d'apprentissage mis en avant dans les pages des revues: plutôt que d'apprendre à coudre ou à cuisiner, les petites filles sont sommées d'apprendre à composer avec des garçons pénibles, incontrôlables, voire dangereux. Si les illustrés sont toujours une presse éducative complétant les enseignements de l'école, ils se font également le relais des difficultés rencontrées dans le cadre scolaire par les petites filles, durant cette période d'après-guerre où le système éducatif connaît des mutations parmi les plus grandes de son histoire. 


\section{L'école est finie?}

se faisaient auparavant dans un cadre domestique et scolaire. L'espace des illustrés pour petites filles reproduisait un environnement familier et familial, avec ses tantes, ses marraines, ses rubriques enseignant l'art et la manière de faire un pot-au-feu ou de tricoter un ensemble écharpe-bonnet pour le petit frère. Il venait remplir les temps libres d'une semaine consacrée à l'école et à des apprentissages transposables au sein $\mathrm{du}$ foyer, selon les principes en cours dans l'enseignement primaire de l'époque, marqué par une idée particulière de la nature féminine ${ }^{29}$. Dans l'après-guerre, les illustrés pour petites filles évoquent davantage un espace semi-public dans lequel évoluent une myriade de personnages qui sont à présent les pairs des lectrices. De verticale, la transmission devient horizontale. Il ne s'agit plus d'apprendre les savoirfaire transmis entre femmes de différentes générations, mais de savoir comment s'intégrer pleinement dans sa classe d'âge. D'école à la maison, le journal s'est fait cour de récréation. Si les petites filles n'échappent pas aux injonctions de genre, elles sont invitées à cohabiter avec les garçons et sont confrontées à l'abandon progressif de la transmission de savoir-faire traditionnels. Elles sont incitées à développer davantage des savoirs non techniques, les compétences liées au «travail émotionnel ${ }^{30}$ " et les savoir-être liés à la consommation et à l'habillement, encouragées dans l'acquisition de ces aptitudes par les regards moqueurs des garçons. Les journaux reproduisent ainsi des dynamiques présentes dans l'enseignement mixte, qui favoriserait un renforcement des stéréotypes de genre par la confrontation des deux sexes ${ }^{31}$.

En 1969, une réforme des rythmes scolaires supprime les cours du samedi après-midi dans l'enseignement primaire. De fait, cela revient souvent à évincer les travaux d'aiguille, qui bénéficiaient de ce créneau dans l'emploi du temps des écolières. L'enseignement ménager laisse ensuite la place à la technologie, pensée comme un enseignement asexués ${ }^{32}$. Dans les périodiques, on observe la lente disparition des rubriques de couture ou de tricot. Les pages des illustrés sont davantage consacrées à deux aspects du temps libre, la consommation et les loisirs. À partir de 1967 arrive dans Lisette et Nade une nouvelle rubrique, qui occupe une place centrale avec quatre pages en couleur richement illustrées: "le journal des cinq"(ill. 9). Chaque semaine, la rubrique est dédiée à la découverte de l'univers d'Emmanuelle, Dominique, Caroline, Dorothée ou Bénédicte, des petites filles avec chacune leur centre d'intérêt et leurs compétences: la nature, les enfants, le sport, le bricolage ou la planification domestique. Si ces appétences restent fortement genrées, ce sont une multiplicité d'identités et d'activités qui sont proposées aux enfants, et non plus un modèle unique de petite fille. Une grande partie des savoir-faire transmis dans cette rubrique relèvent des loisirs, ou présentent des tâches domestiques sous un angle ludique. Les travaux d'aiguille deviennent une activité parmi d'autres, pour des petites filles qui ne sont plus définies dans leur rapport à une norme univoque, mais davantage grâce à leurs capacités à articuler les différentes facettes d'une personnalité multidimensionnelle, partagée entre l'école, les loisirs, le sport, les relations familiales et amicales. 
III.9. « Le journal des Cinq : Dorothée et son aquarium », Lisette, 17 novembre 1968. Droits réservés.
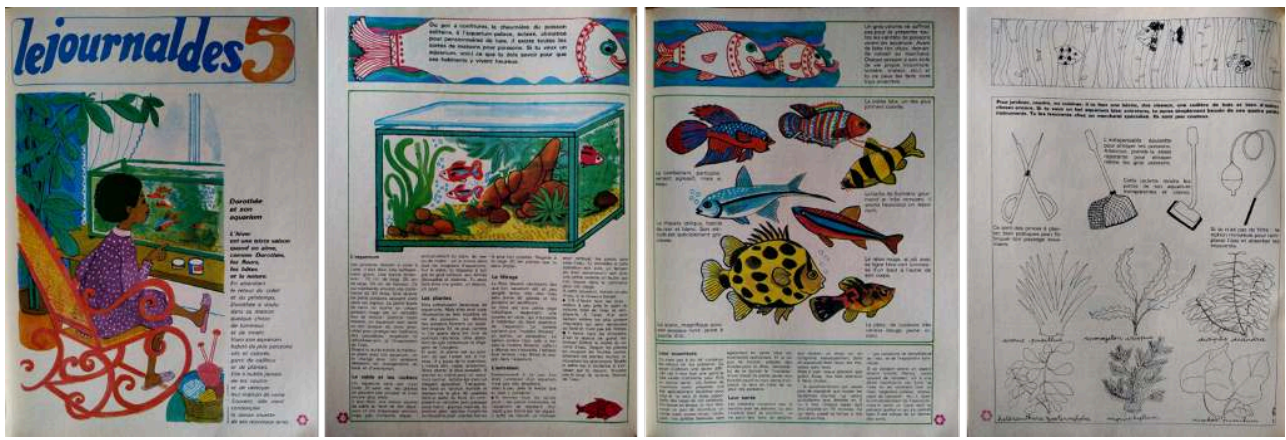

Les périodiques témoignent ainsi d'un temps relativement libéré des contraintes parentales, à l'origine d'une nouvelle sociabilité et de modes de vie spécifiques qui se développent alors, jusqu'à constituer une nouvelle classe d'âge, l'adolescence. Ces représentations relevant de la " culture jeune " permettent en réalité à ces journaux de continuer à intéresser la portion la plus âgée de leur lectorat, en les projetant dans des modes de vie moins contraints qui ne sont pas encore les leurs. Les jeunes lectrices ont en effet tendance à se reporter facilement sur les magazines destinés à leurs aînées, quand bien même elles n'en constituent pas le public cible; Jackie, hebdomadaire britannique destiné aux 15-20 ans apparu dans les années 1960, était couramment lu par des filles de 11 à 13 ans $^{33}$. Mademoiselle Âge Tendre, publication mensuelle à grand tirage lancée en 1964, représente une menace forte pour les périodiques pour petites filles, qui voient leur public habituel capté par des journaux destinés aux plus âgées. Les illustrés ne conservent leur popularité jusqu'à la fin des années 1970 qu'auprès des plus jeunes, les 7-11 ans restant des consommateurs substantiels de ces hebdomadaires ${ }^{34}$.

\section{Salut les copines !}

Dans les années 1960 et 1970, les hebdomadaires pour petites filles sont de plus en plus confrontés à des problèmes commerciaux. Ces périodiques, jusqu'alors d'une grande stabilité de maquette comme de contenus, multiplient les tentatives pour cacher la crise qu'ils traversent et reconquérir un public s'effritant peu à peu. Après sa réunion avec Nade, Lisette changera ainsi trois fois de propriétaire et de nom entre 1970 et 1974, et sa fusion finale avec $M^{\text {lle }}$ Caroline apportera son lot d'innovations graphiques étranges avec des adaptations illustrées de séries télévisées reprenant les personnages de Caroline et ses compagnons félins (ill. 10) ${ }^{35}$. 
III.10. « Devine qui est derrière la page ? ", Lisette et Caroline, Société Française de Presse Illustrée, 15 mai 1973, p. 34. Droits réservés.

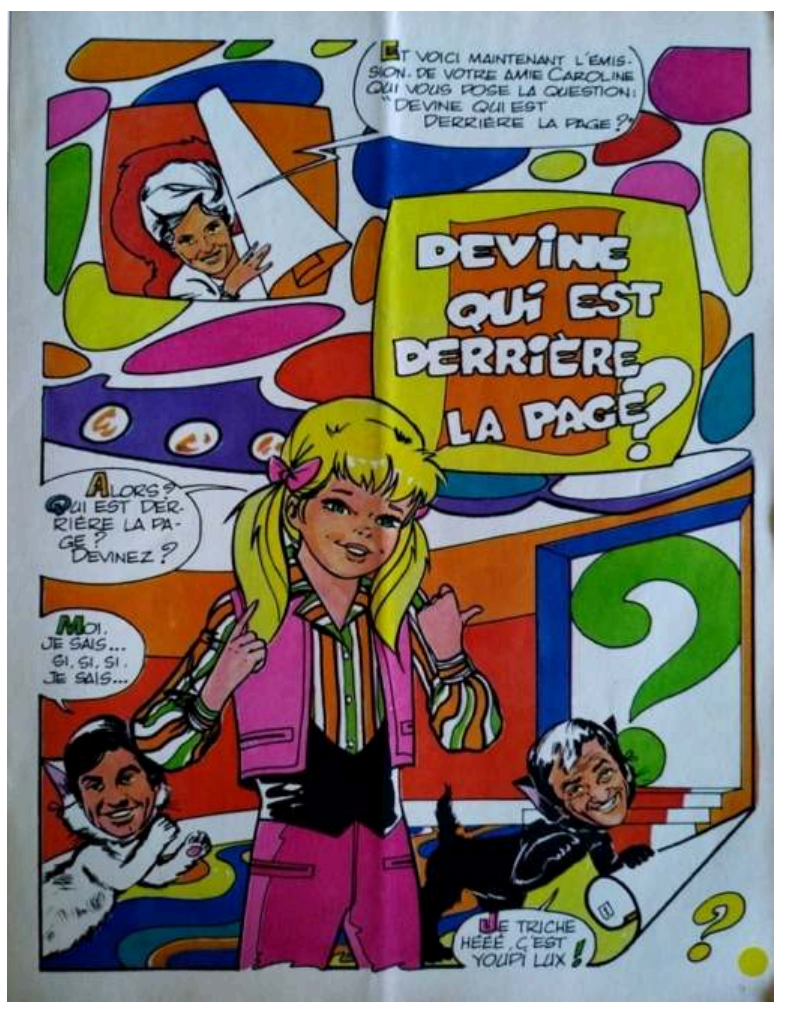

Dans les années 1970, l'illustré pour petites filles, en tant qu'outil éducatif appuyé sur des principes pédagogiques et relais des discours institutionnels, n'avait plus d'objet. Dans un contexte de valorisation et de généralisation de la mixité scolaire qui impose la vision d'un élève neutre, ces hebdomadaires ont eux-mêmes sonné leur glas : comment justifier leur existence tout en se faisant le relais des aspirations à l'universalisme du système scolaire? Suzette, Lisette, Bernadette et Fillette ont contribué pendant les années d'après-guerre à ancrer dans les mentalités la pensée d'une nécessaire complémentarité des deux sexes dans le cadre des apprentissages primaires et secondaires. À une époque où les féministes de la seconde vague s'interrogent sur l'instauration d'espaces de non-mixité dans le cadre du militantisme, le discours porté par l'institution scolaire et relayé par les illustrés pour petites filles tente de naturaliser un processus récent et peu débattu dans l'arène politique de l'après-guerre. Avec la généralisation de la mixité scolaire est confirmée la vision d'une enfance comme période d'indifférenciation sexuelle. Bayard Presse, avec son large éventail de titres mixtes pour la jeunesse, embrasse cette idéologie, en proposant une panoplie de journaux conjugués au "neutre masculin ${ }^{36}$ ", au risque de rendre "moins visible et moins compréhensible la marginalisation féminine ${ }^{37}$ ». 
III.11. "Le journal des Cinq : Caroline a une nouvelle passion », Lisette Télé Jeune, Éditions de Montsouris, 11 mars 1973, p. 10-11. Droits réservés.

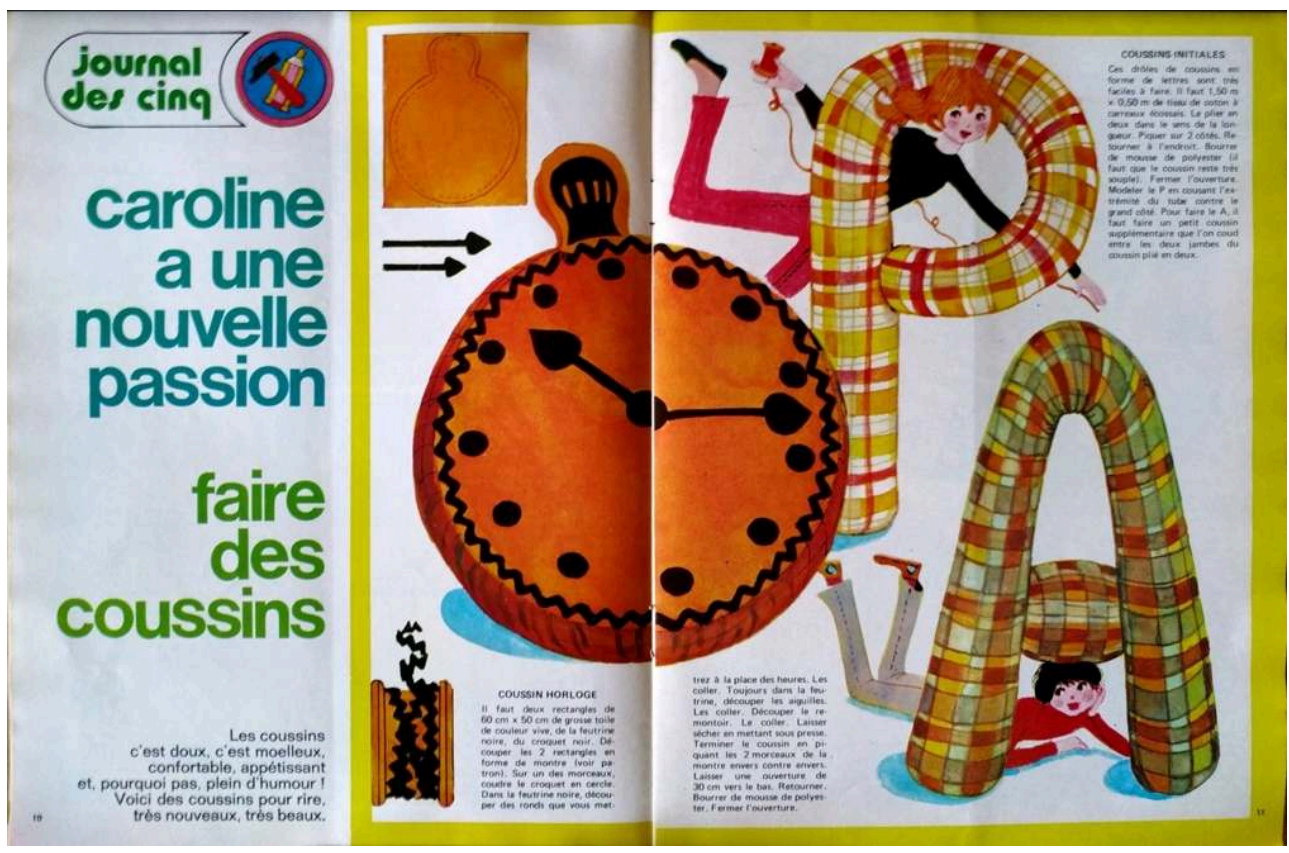

Après les années 1970, les magazines féminins subsistent et les jouets renvoyant aux tâches ménagères continuent d'être dévolus aux petites filles. Si le journal pour fillettes a disparu, c'est parce qu'il n'est ni un magazine féminin, ni un jouet, mais le porteur d'un fort rôle moral et d'un discours institutionnel. La disparition des périodiques pour petites filles documente également la rupture des liens de transmission familiale et féminine, et la perte des savoir-faire techniques au profit de compétences liées à la consommation. Tricoter et broder ne s'inscrivent plus dans les apprentissages indispensables à la petite fille pour participer à l'économie domestique, mais rejoignent le rang des loisirs qui participeraient à l'épanouissement individuel des fillettes (ill. 11). Dans le même temps, les hebdomadaires pour petites filles continuent de proposer des activités associées à leur genre, et gardent la fonction socialisatrice associée à l'ensemble de la presse féminine jeunesse de l'époque selon Marie Duru-Bellat: «maintenir les femmes dans l'exercice de leur rôle social traditionnel ${ }^{38}$ ». Tandis que la réalisation de petits bricolages et les travaux manuels deviennent l'apanage des plus jeunes lectrices ${ }^{39}$, les plus âgées reçoivent de fortes injonctions à savoir choisir leur habillement avec goût, écouter la musique à la mode ou encore danser. Les compétences liées à la communication sont particulièrement valorisées: il s'agit d'avoir des amies, peut-être des prétendants, tout en respectant un ensemble de règles intangibles que les jeunes adolescentes doivent apprendre à maîtriser. Par ailleurs, la mécanisation des tâches ménagères libère du temps dans l'agenda des adolescentes, temps que Luce Giard qualifie de " gris, vide, homogène, le temps de l'ennui sans effort et sans joie ${ }^{40} »$. Avant les années 1950, il s'agissait de s'inscrire dans une structure patriarcale et une organisation familiale qui proposaient des transmissions fortes intergénérationnelles. Avec la disparition des Tante Marmelade, Tante Conseil et autres marraines, les illustrés des années 1960 voient se taire «le peuple féminin des cuisines $»^{41}$, au profit d'un omniprésent jugement des pairs sur les qualités physiques et morales de leurs petites camarades de classe. Autrefois rappelées à l'ordre par les figures tutélaires du journal lorsqu'elles ne remplissaient pas correctement leur rôle social, 
elles le sont à présent par des garçons qui leur reprochent d'être trop grosses ou de jouer au football. Comment devenir une "chic fille»? À quel moment est-on une " pimbêche » ou un "garçon manqué »? Les périodiques pour petites filles des années 1960 rendent compte de ce déchirement entre deux publics jusqu'alors pensés comme homogènes : d'un côté des petites filles désormais supposées recevoir une formation identique à celles des garçons, de l'autre des adolescentes qui, confrontées à leurs changements corporels, doivent intégrer des compétences et des parcours conformes à leur genre. La neutralité devient une caractéristique des titres enfantins, tandis que la presse féminine propose de nouveaux titres adaptés aux très jeunes filles. Le secteur de l'édition s'adapte ainsi aux injonctions nouvelles adressées aux lectrices dans un système qui maintient les oppressions tout en les camouflant durant l'enfance par un "neutre masculin", dont la visée universalisante est aujourd'hui une nouvelle fois contestée par des mouvements féministes en quête de non-mixité.

\section{NOTES}

1. Jessica Kohn, "Travailler dans les Petits Mickeys": les dessinateurs-illustrateurs en France et en Belgique de 1945 à 1968, thèse de doctorat en histoire, sous la direction de Jean-Paul Gabilliet et Laurent Martin, Université Sorbonne Paris Cité, 2018, p. 727.

2. Les illustrés pour petites filles sont explicitement destinés aux 7-15 ans. Cette tranche d'âge est plus large que celles adoptées aujourd'hui dans le cadre des politiques de chaînage des journaux enfantins. Il est impossible de désigner les lectrices de ces hebdomadaires d'une appellation unique. J'ai donc opté pour les termes « petites filles » et « fillettes », employés couramment dans le rédactionnel des journaux.

3. Voir Sylvette Giet, "Lisette, un illustré entre tradition et modernité (1937-1939) " et Manon Pignot, «Suzette contre Fillette : la Grande Guerre de deux illustrés français », dans Thierry Crépin et Françoise Hache-Bissette (dir.), Les Presses enfantines chrétiennes au $\mathrm{Xx}^{e}$ siècle, Arras, Artois Presses Université, 2008, respectivement p. 77-90 et p. 211-224; Marie-Anne Couderc, La Semaine de Suzette. Histoire de filles, Paris, CNRS éditions, 2005.

4. Yves-Marie Cloître, "Salut les copains ", dans Claude Dufrasne (dir.), Des millions de jeunes. Aspects de la jeunesse, Paris, Éditions Cujas, 1967, p. 119-149; Anne-Marie Sohn, Âge tendre et tête de bois. Histoire des jeunes des années 1960, Paris, Hachette Littératures, 2001 ; Alain Vergnioux et JeanMarc Lemonnier, «Les adolescents des années soixante : salut les copains! », Le Télémaque, vol. 2, $\mathrm{n}^{\circ} 38,2010$, p. 87-100. Disponible sur Cairn [En ligne], mis en ligne le 24 février 2011, consulté le 28 mai 2021, URL : https://www.cairn.info/revue-le-telemaque-2010-2-page-87.htm.

5. Jean-Yves Mollier, «La naissance de la culture médiatique à la Belle-Époque : mise en place des structures de diffusion de masse ", Études littéraires, vol. 30, $n^{\circ}$ 1, 2005, p. 18.

6. Alain Fourment, Histoire de la presse des jeunes et des journaux d'enfants, 1768-1988, Paris, Éditions Eole, 1987, p. 88.

7. Annie Renonciat, «Quand la chambre fait école. Images et usages pédagogiques de la chambre d'enfant ", Strence [En ligne], 2014, $\mathrm{n}^{\circ} 7$, mis en ligne le $1^{\mathrm{er}}$ juin 2014, consulté le 2 novembre 2020, URL : http://journals.openedition.org/strenae/1233.

8. Annie Renonciat, «De l'Orbis sensualium pictus (1658) aux premiers albums du Père Castor (1931) : formes et fonctions pédagogiques de l'image dans l'édition française pour la jeunesse », 
dans Annie Renonciat et Marianne Simon-Oikawa (dir.), La Pédagogie par l'image en France et au Japon, Rennes, Presses universitaires de Rennes, 2009, p. 66-67.

9. Françoise Hache-Bissette, "Chapitre 12. La presse pour la jeunesse : entre éducation et récréation », dans Claire Blandin (dir.), Manuel d'analyse de la presse magazine, Paris, Armand Colin, 2018, p. 204.

10. Alain Fourment, Histoire de la presse des jeunes et des journaux d'enfants, op. cit., p. 153.

11. Françoise Hache-Bissette, op. cit., p. 208.

12. Annie Renonciat, «Quand la chambre fait école [...]», art. cit.

13. Sylvain Lesage, "Apprendre et consommer, apprendre à consommer. Image d'Épinal et pédagogie publicitaire », Le Magasin du XIX siècle, nº 5, 2016, p. 92.

14. Claire Le Thomas, "Une littérature du foyer : les livres de travaux manuels amateur ", Voix plurielles, vol. $5, \mathrm{n}^{\circ} 1,2008$, p. 3.

15. Joël Lebeaume, L'Enseignement ménager en France. Sciences et techniques au féminin, Rennes, Presses universitaires de Rennes, 2014.

16. Fillette est dénoncée par les censeurs au $\mathrm{xx}^{\mathrm{e}}$ siècle car elle est publiée par les Frères Offenstadt, qui s'étaient jusqu'alors illustrés dans le domaine des publications érotiques ou relevant d'un humour grivois. D'origine allemande et de confession juive, ils sont régulièrement l'objet d'attaques antisémites.

17. Alain Fourment, Histoire de la presse des jeunes et des journaux d'enfants, op. cit., p. 92.

18. Colette Guillaumin, "Pratique du pouvoir et idée de Nature (1). L'appropriation des femmes ", Questions féministes, ${ }^{\circ}$ 2, 1978, p. 7.

19. Joël Lebeaume, L'Enseignement ménager en France, op. cit.

20. Françoise Thébaud, «La nationalisation des femmes », dans Georges Duby, Michelle Perrot et François Thébaud (dir.), Histoire des femmes en Occident. Tome 1, Paris, Plon, 1992, p. 24-235.

21. Joël Lebeaume, L'Enseignement ménager en France, op. cit.

22. Véronique Blanchard, Vagabondes, voleuses, vicieuses, adolescentes sous contrôle, de la Libération à la libération sexuelle, Paris, Éditions François Bourin, coll. « Genre !», 2019, p. 37.

23. Alain Tirefort, «Les petites Suzette aux colonies. La Semaine de Suzette et la culture coloniale pendant l'entre-deux-guerres ", Afrika Zamani, n 9-10, 2001-2002, p. 104 ; Marie-Anne Couderc, La Semaine de Suzette, op. cit., p. 42.

24. Anne-Marie Sohn, «Les "relations filles-garçons" : du chaperonnage à la mixité (1870-1970) », Travail, genre et sociétés, vol. 9, $\mathrm{n}^{\circ}$ 1, 2003, p. 103 ; Michelle Zancarini-Fournel, "Coéducation, gémination, co-instruction, mixité : débats dans l'Éducation nationale (1882-1976)», dans Rebecca Rogers (dir.), La Mixité dans l'éducation : enjeux passés et présents, Lyon, ENS éditions, 2004, p. 35.

25. Michelle Zancarini-Fournel, « Coéducation, gémination, co-instruction, mixité [...]», art. cit., p. 33.

26. Lisette passe de 234000 exemplaires tirés en 1951 à 135000 en 1956 ; Fillette sur la même période connaît une baisse de tirage de 30000 exemplaires, alors qu'elle n'en éditait que 137500 en 1951. Enfin, Bernadette divise par deux ses tirages en quelques années, passant de 198000 exemplaires en 1953 à 93985 en 1958. Cette baisse se confirmera dans les années 1960. Alain Fourment, Histoire de la presse des jeunes et des journaux d'enfants, op. cit., p. 411-412.

27. Françoise Hache-Bissette, «Bayard et Milan : deux marques concurrentes de presse éducative au sein d'un même groupe ", Le Temps des médias, n² 21, automne-hiver 2013, p. 54.

28. Traduction personnelle : "Il existe une tension évidente entre le besoin de confirmer que la présence des hommes doit être centrale et désirable dans la vie des femmes et le fait de reconnaître avec une même intensité que les hommes sont un problème et une menace pour les femmes ». Ros Ballaster, Margaret Beetham, Elizabeth Frazer, et Sandra Hebron, Women's Worlds. Ideology, feminity and the woman's magazine, New York, MacMillan Press Ltd, 1991, p. 19.

29. Joël Lebeaume, L’Enseignement ménager en France, op. cit. 
30. Arlie Russell Hochschild, "Travail émotionnel, règles de sentiments et structure sociale », Travailler, vol. 9, $\mathrm{n}^{\circ} 1,2003$.

31. Marie Duru-Bellat, L'École des filles. Quelle formation pour quels rôles sociaux ?, Paris, L'Harmattan, coll. « Bibliothèque de l'éducation », 2004, p. 75.

32. Joël Lebeaume, L'Enseignement ménager en France, op. cit.

33. Ros Ballaster et al., Women's Worlds, op. cit., p. 11.

34. Alain Fourment, Histoire de la presse des jeunes et des journaux d'enfants, op. cit., p. 339.

35. Michelle Daufresne, illustratrice à la Semaine de Suzette, donne un aperçu du fonctionnement chaotique des dernières années du journal, qui vont l'amener jusque dans les bureaux de la police judiciaire pour témoigner de détournements d'argent survenus sur fond d'histoire de drogue à la rédaction du journal. Jessica Kohn, «"Travailler dans les Petits Mickeys », op. cit., p. 727.

36. Sylvie Cromer, « Le masculin n'est pas un sexe : prémices du sujet neutre dans la presse et le théâtre pour enfants ", Cahiers du genre, vol. 49, n 2, 2010, p. 99.

37. Ibid., p. 112.

38. Marie Duru-Bellat, L'École des filles, op. cit., p. 109.

39. Catherine Monnot, Petites filles d'aujourd'hui. L'apprentissage de la féminité, Paris, Éditions Autrement, p. 81-82.

40. Luce Giard dans Michel de Certeau, Luce Giard et Pierre Mayol (dir.), L'Invention du quotidien, tome 2 : Habiter, cuisiner, Paris, Gallimard, 1994, p. 299.

41. Ibid., p. 219.

\section{RÉSUMÉS}

À partir du début du xx siècle et jusque dans les années 1970, les illustrés pour petites filles connaissent une grande popularité. La Semaine de Suzette, Fillette, Bernadette et Lisette proposent chaque semaine à leurs lectrices de nombreuses rubriques destinées à les former à leur futur rôle social de mère et d'épouse. Dans leur forme et leur contenu, ces titres prolongent et complètent l'enseignement ménager dispensé dans l'enseignement primaire et secondaire. Ils apparaissent comme un support éducatif, qui recoupe une vision différencialiste des sexes portée par l'État. Après-guerre, la généralisation de la mixité complexifie le rôle des périodiques, qui dans la lignée de leur mission éducatrice se retrouvent à porter un discours institutionnel pro-mixité qui entre en contradiction avec leur existence même. Confrontés à une instruction publique prônant une éducation unique, les hebdomadaires pour petites filles laissent la place à des publications mixtes, dans un contexte de rupture des transmissions intergénérationnelles féminines.

\section{INDEX}

Mots-clés : illustration, magazines pour la jeunesse, stéréotypes féminins, mixité, apprentissage 
AUTEUR

BÉATRICE GUILLIER

Doctorante à l'École des hautes études en sciences sociales

Laboratoire : IIAC (EHESS/CNRS)

Chercheuse associée à la Bibliothèque nationale de France, au Centre national de la Littérature pour la Jeunesse

beatrice.guillier@ehess.fr 